\title{
ACCURACY OF THE CONVERGENT PHOTOGRAPHY
}

By

Dr. Abo El-Hassan M. Rahil

Faculty of Eng. Schebin El - Kom, Minufiya University, Egypt

\begin{abstract}
There is no doubt that the number and variety of the photogrammetric applications are growing. Convergent case photogrammetry is the most commonly used in analytical photogrammetry. The present formulas which are used to estimate the accuracy of convergent case photogrammetry do not give good results, Special at the edge of photograph therefor, the simulation is used for estimating the accuracy of the convergent case of close range photogrammetry. The accuracy obtained in practical experiments closely matches the estimated accuracy from simulation. The disadvantages of the simulation are :

The method needs an experience and skilled photogrammetrist;

The optimal solution is missed and;

The simulation technique is costly and complicated.

This paper gives the mathematical proofs of a newly developed formulas for estimating the accuracy of convergent case.
\end{abstract}

Manuscript received from Dr; Abou El Hasssan Rahil on : $30 / 5 / 1999$

Accepted on: 24/8/1999

Engineering Research Bulletin, Vol 22,No 3, 1999

Minufiya University, Faculty of Engineering, Shebin El-Kom, Egypt, ISSN 1110-1180 


\section{INTRODUCTION}

The problem which faces the photogrammetrist is in determining the optimum positions of the two camera stations relative to the object in order to achieve the required accuracy. So far, there have been no satisfactory mathematical formulas, in the field of photogrammetry, which allow. the photogrammetrist to determine the optimum layout of the two cameras stations. The main objective of this study is to find an ideal solution for the accuracy problem by devloping formulas which make it possible for the photogrammertist to determine the optimum layout of the two cameras stations and also to determine the object accuracy, for any camera set-up, right in the field. The accuracy of the ground coordinates is a function of the elements of the photogrammetric system. The principal parameters influencing acuuracy may be classified into three groups :

\section{A-Geometrical charateristics:}

The accuracy depends on the lay out of the two camera stations; number of stations; density of control and the foacl length of the camera. The layout of the two camera stations relative to the object is a function of the external orientation parameters. The accuracy of the object - space coordinates obtained from convergent photographs is a function of these parameters $Y, B, C, \varphi, \sigma_{x}$, $\sigma_{\mathrm{z} \cdot \text {. }}$

These parameters can be classified into two groups:

\section{Configuration parameters :}

$B ; Y$ and $\varphi$ which change by changing the outer oriention of the two photos. where

$\mathrm{B}$ : is the base between the two photos,

$\mathrm{Y}:$ is the mean object distance,

$\varphi$ : is the angle of convergence.

2. Camera parameters:

$\mathrm{C} ; \sigma_{\mathrm{x}} ; \sigma_{\mathrm{z}}$ and the formate size.

where

$\mathrm{C}$ : is the camera constant,

$\sigma_{\mathrm{x}}, \sigma_{\mathrm{z}}$ : are the standard errors of the image coordinates $\mathrm{x}$ and $\mathrm{z}$ respectively.

- B- Physical characteristics of the photogrammetric system:

- The principal physical characteristics of the photogrammetric system are:

- The quality of the camera objectives and the lens distortions; The quality of the film ;The definition of the object ( natural or artificial) and The accuracy of comparator measurements.

\section{C- Redundance of measurements:}

There are three faces for the redundance of measurements :

The number of stations and number of frames at each station; number of comparator measurements for each point for each photo and number of targets for each object point . 


\section{PREDICTION OF ACCURACY}

Accuracy predictors are formulas (Abdel Aziz and Karara)[1], simulations and diagrames (Hottier)[6], which give the accuracy as a function of the principal parameters of a photogrammetric system. Accuracy predictors can exist only for simple configurations of the data acquisition system (the symmetric case of the pair ) which are the most frequent in practice .

The existing formulas for convergent photogrmmetry case express the accuracy of ground points as a function of the configuration parameters $Y, B, \varphi$ of the two photos that form the model, This formulas according to Abdel Aziz and Karara [1], which give the errors $\left(\sigma_{\mathrm{X}}, \sigma_{\mathrm{Y}}\right.$ and $\sigma_{\mathrm{Z}}$ ) ( X- axis parallel to the base,and $\mathrm{Y}$ axis perpendicular to the object Figure 1 ) reffered to the image plane are:

$$
\begin{aligned}
\sigma_{X} & =\frac{Y}{C} \frac{(1+\tan \alpha \tan \varphi) \sigma}{1-\tan (\alpha-\varphi) \tan \varphi} \\
\sigma_{Y} & =\frac{Y}{C} \frac{\sec \varphi \sigma}{1-\tan (\alpha-\varphi) \tan \varphi} \\
\sigma_{Z} & =\frac{Y}{C} \frac{Y}{B} \sqrt{2} \frac{(1+\tan \alpha \tan \varphi) \sigma}{1-\tan (\alpha-\varphi) \tan \varphi}
\end{aligned}
$$

where:

$B$ : is the base between the two photos,

$Y$ : is the mean object distance,

$\sigma$ is the standard deviation of the measurement error,

$\varphi \quad$ is the angle which the camera axis makes with the direction perpendicular to the base

$\alpha$ is the angle which the line joining the central point $O$ of the object space and the perspective centre makes with the perpendicular direction to the base.

The computation of the accuracy of the object space coordinates is based on the following assumptions :

a- $\sigma_{\mathrm{x} 1}=\sigma_{\mathrm{x} 2}=\sigma_{\mathrm{z} 1}=\sigma_{\mathrm{z2}}=\sigma$

b- $\varphi_{1}=\varphi_{2}=\varphi, \alpha_{1}=\alpha_{2}=\alpha$ 


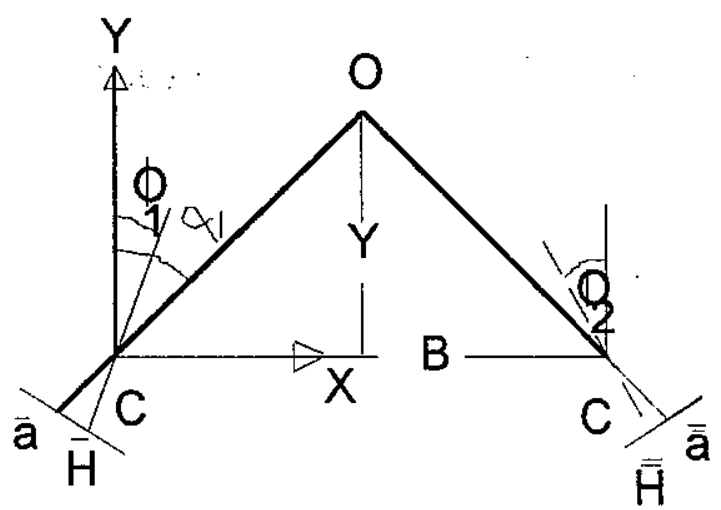

Fig.1 The covergent case of photogrammetry. Karara and Abdel Aziz [2], have studied the accuracy problem by considering the accuracy only at the centeral object point, where the implicit assumption being that the accuracy at the central object point can reasonably represent the accuracy of the whole object . Hottier [6], has found that the central point gives theoretically a slight lower accuracy . Hottier [6], and Regensburger[8], have concluded that the accuracy at the central point cannot represent in an ideal way the average accuracy over the whole object plane, particularly for small values of the base- to - object - distance ratio $\left(0.7<\mathrm{r}^{1}<1.0\right)$. They have found that the central point $\mathrm{XY}$ accuracy is of course constant, but it is not the same for corner points .

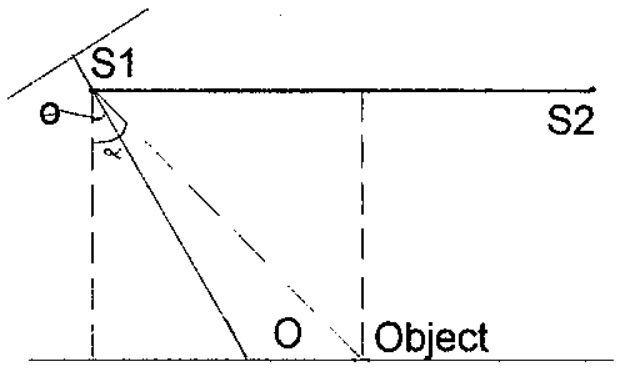

Fig.2. Definition of the central point [6].

This means that these formulas cannot give the real accuracy over the whole object specially at the corner points. Accordingly, Hottier used the simulation technique for estimating the accuracy of the object points. The estimated accuracy by simulations yields results which closely match the accuracy obtained from close-range applications. The simulation technique cannot be recommended for real applications because it is costly and complicated specially for the error model .Moreover, the photogrammetrist cannot estimate the ground accuracy immediately in the field for any camera stations. Accordingly, new formula have been developed in this article expressing the accuracy of the ground points over the whole object as a function of the above mentioned parameters of external oriention. These formulas can be used in the field for determining the positions of the two camera stations and for computing the accuracy of the object points.

${ }^{i} \mathrm{r}=\mathrm{B} / \mathrm{H}$ 


\section{THE THEORETICAL ASPECT}

Figure 3 shows two coordinate system $X, Y$ and $X_{1}, Y_{1}$ having the same origin $O$, but the coordinate system $X_{1}, Y_{1}$ is rotated throught an angle $\varphi$ relative to the fixed coordinate system $X, Y$. In this figure, the camera axis (CA) coincide to the $Y_{1}$ direction and $X_{1}$ is perpendicular to the $Y_{1}$ direction, so the camera axis (CA) and its perpendicular axis $X_{1}$ represent the coordinate system $X_{1}, Y_{1}$. According to the position of point $A$ with respect to $Y_{1}$ direction one have 3 different cases.

Case 1 : Point $A$ lies between camera axis $(C A)\left(Y_{1}\right.$ - direction $)$ and $X$-axis , in this case $\alpha>\varphi$,

Case 2: Point A lies between camera axis (CA) $\left(Y_{1}\right.$ - direction ) and $Y$-axis, in this case $\varphi>\alpha$,

Case 3: Point $\mathrm{A}$ lies on the direction of the camera axis, in this case $\varphi=\alpha$.
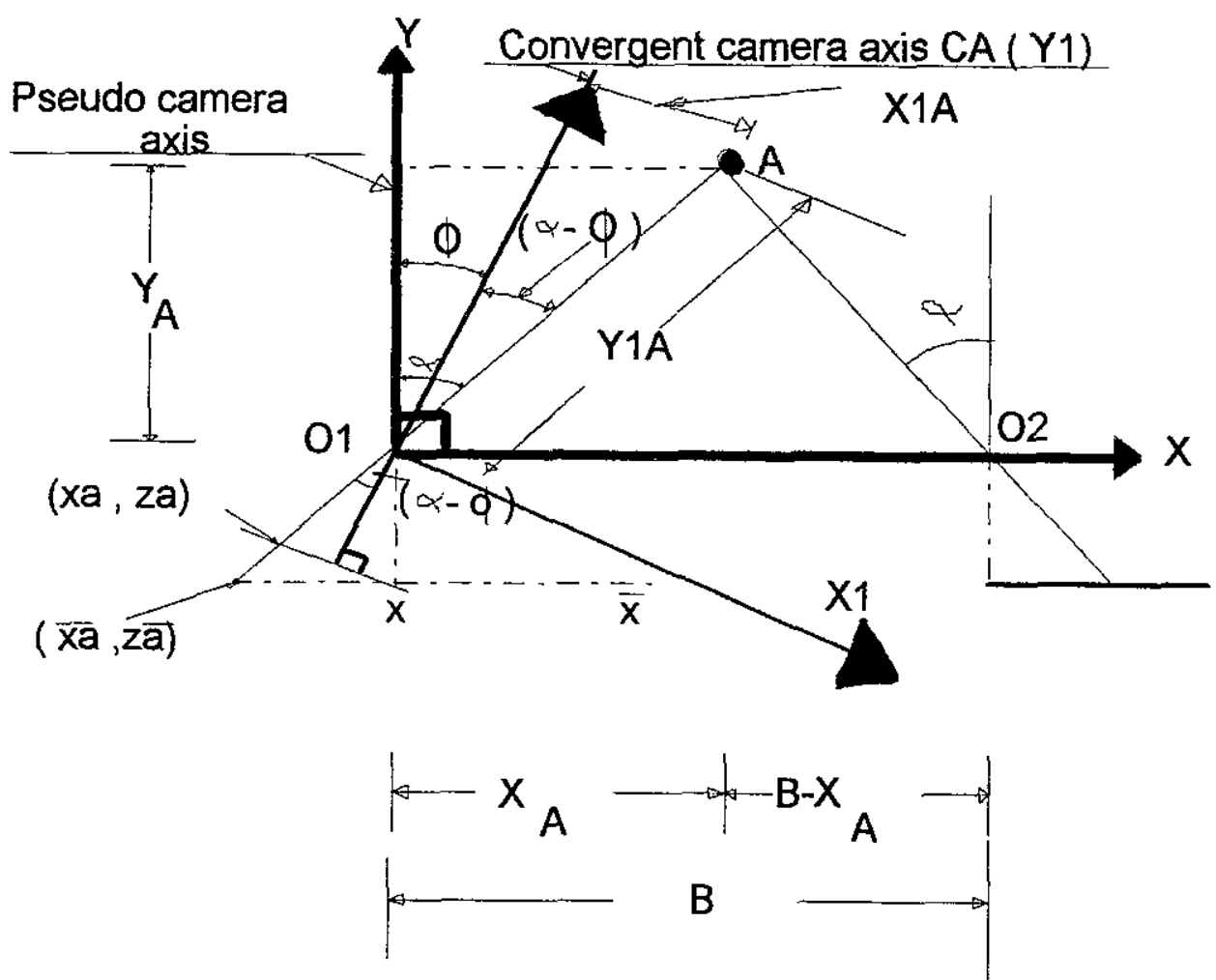

Fig.3 The relationsh between the ground coordinates of the same point $A$ on a convergent photo on two coordinate system ( $X, Y$ and $Z$ and $\mathrm{X} 1, \mathrm{Y} 1$ and $\mathrm{Z} 1$ ). 


\section{Case 1: Point $A$ lies between camera axis (CA) and the $X$-axis}

According to Figure 3 the relationship between the ground coordinates of the same point $A$ on two different coordinate systems $X, Y$ and $X_{1}, Y_{1}$ can be deduced as follows:

$$
\begin{aligned}
& X_{1 A}=X_{A} \cos \varphi-Y_{A} \sin \varphi \\
& Y_{1_{A}}=X_{A} \sin \varphi+Y_{A} \cos \varphi
\end{aligned}
$$

or in matrix form :

$$
\left(\begin{array}{l}
X_{l_{A}} \\
Y_{1_{A}}
\end{array}\right)=\left(\begin{array}{cc}
\cos \varphi & -\sin \varphi \\
\sin \varphi & \cos \varphi
\end{array}\right)\left(\begin{array}{l}
X_{A} \\
Y_{A}
\end{array}\right)
$$

From Fig. 3 one find :

$$
\begin{aligned}
& \frac{\bar{x}}{C}=\frac{X_{A}}{Y_{A}}=\tan \alpha \\
& \frac{x}{C}=\frac{X_{1 A}}{Y_{1}}=\tan (\alpha-\varphi)
\end{aligned}
$$

by substituting from equation 4 , into equation 6 one can gets :

$$
\frac{x}{C}=\frac{X_{1}}{Y_{1}}=\tan (\alpha-\varphi)=\frac{X \cos \varphi-Y \sin \varphi}{X \sin \varphi+Y \cos \varphi}
$$

According to Abdel Aziz [2]/p. 1344 the accuracy of the image coordinates are :

$$
\begin{aligned}
\sigma_{x}= & \frac{\left(1+\left(\frac{x}{C}\right) \tan \varphi\right) \sigma}{1-\left(\frac{x}{C}\right) \tan \varphi} \\
\sigma_{y}= & \frac{\sec \varphi \sigma}{1-\left(\frac{x}{C}\right) \tan \varphi}
\end{aligned}
$$

By substituting from equations 5 and 7 into equations 8 and 9 one finds that, the accuracy of the image coordinates of any point $A$ lies between the camera axis (CA) and $X$ axis are :

$$
\sigma_{x}=\sigma_{x 1}=\sigma_{x 2}=\frac{(X \sin \varphi+Y \cos \varphi)^{2}}{Y^{2}}
$$




$$
\sigma_{y}=\sigma_{y 1}=\sigma_{y 2}=\frac{(X \sin \varphi+Y \cos \varphi)}{Y}
$$

\section{THEORETICAL ACCURACY OF THE OBJECT-SPACE COORDINATES}

Karara[7],deduced that the accuracy of the object space coordinates $X, Y$ and $\mathrm{Z}$ in the normal case of photogrammetry reffering to Fig (4) are :

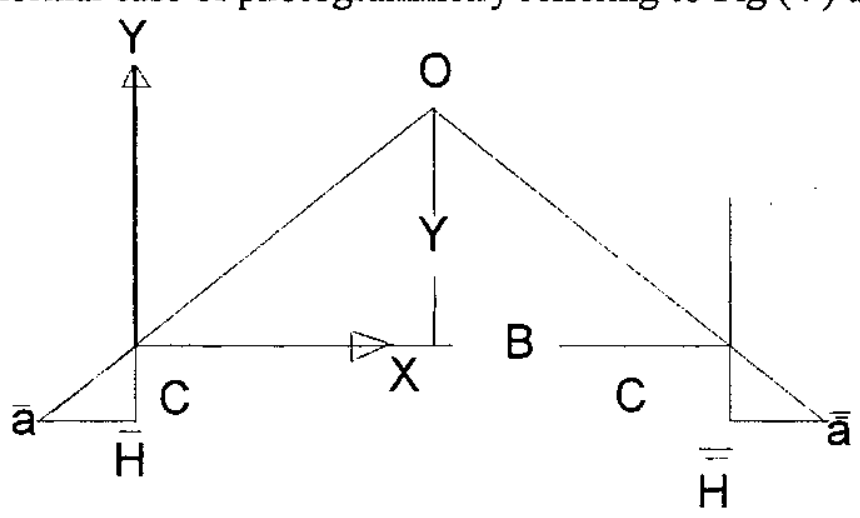

Fig. 4. The normal case of photogrammetry.

$$
\begin{gathered}
\sigma_{X}=\left(\frac{Y}{C}\right) \sigma_{x} \\
\sigma_{Z}=\left(\frac{Y}{C}\right) \sigma_{z} \\
\sigma_{Y}=\frac{Y}{C} \frac{Y}{B} \sqrt{2} \sigma_{x}
\end{gathered}
$$

Substituting the values of $\sigma_{x}, \sigma_{y}$ from equations 10 and 11 into equations 12 and 13 and 14 one gets :

$$
\begin{gathered}
\sigma_{X}=\frac{1}{C} \frac{1}{Y}(X \sin \varphi+Y \cos \varphi)_{\sigma}^{2} \\
\sigma_{Z}=\frac{1}{C}(X \sin \varphi+Y \cos \varphi)_{\sigma} \\
\sigma_{Y}=\frac{1}{C} \frac{1}{B} \sqrt{2}(X \sin \varphi+Y \cos \varphi)_{\sigma}^{2}
\end{gathered}
$$


Case 2: Point $A$ lies between camera axis (CA) and $Y$-axis (in this case $\varphi>\alpha$ )

According to Figure 3 the relationship between the ground coordinates of the same point $A$ on two different coordinate system $X, Y$ and $X_{1}, Y_{1}$ can be deduced as follows:

$$
\begin{aligned}
& X_{1}=-X \cos \varphi+Y \sin \varphi \\
& Y_{1}=X \sin \varphi+Y \cos \varphi .
\end{aligned}
$$

or in matrix form :

$$
\left(\begin{array}{l}
X_{1} \\
Y_{1}
\end{array}\right)=\left(\begin{array}{ll}
-\cos \varphi & \sin \varphi \\
\sin \varphi & \cos \varphi
\end{array}\right)\left(\begin{array}{l}
X \\
Y
\end{array}\right)
$$

From Fig. 3

$$
\begin{array}{r}
\frac{\bar{x}}{C}=\frac{X}{Y}=\tan \alpha \quad(19) \\
\frac{x}{C}=\frac{X_{1}}{Y_{1}}=\tan (\varphi-\alpha)
\end{array}
$$

by substituting from equation 18 , into equation 20 one can gets :

$$
\frac{x}{C}=\frac{X_{1}}{Y_{1}}=\tan (\varphi-\alpha)=\frac{-X \cos \varphi+Y \sin \varphi}{X \sin \varphi+Y \cos \varphi}
$$

By substituting the values of $\left(x^{*} / C\right)$ and $(x / C)$ from equations 19 and 21 into equations 8 and 9 one finds that, the accuracy of the image coordinates of any point $\mathrm{A}$ lies between the camera axis $(\mathrm{CA})$ and $\mathrm{Y}$ axis are :

$$
\begin{aligned}
& \sigma_{x}=\sigma_{x 1}=\sigma_{x 2}=\frac{1}{Y} \frac{(X \sin \varphi+Y \cos \varphi)^{2}}{\sin \varphi \cos \varphi(Y+X)+Y\left(\cos ^{2} \varphi-\sin ^{2} \varphi\right)} \\
& \sigma_{y}=\sigma_{y 1}=\sigma_{y 2}=\frac{(X \sin \varphi+Y \cos \varphi)}{\sin \varphi \cos \varphi(Y+X)+Y\left(\cos ^{2} \varphi-\sin ^{2} \varphi\right)}
\end{aligned}
$$


Substituting the values of $\sigma_{\mathrm{x}}$ and $\sigma_{\mathrm{y}}$ from equations 22 and 23 into equations 12,13 and 14 one get:

$$
\begin{gathered}
\sigma_{X}=\frac{1}{C} \frac{(X \sin \varphi+Y \cos \varphi)^{2} \sigma}{\sin \varphi \cos \varphi(Y+X)+Y\left(\cos ^{2} \varphi-\sin ^{2} \varphi\right)} \\
\sigma_{Z}=\frac{Y}{C} \frac{(X \sin \varphi+Y \cos \varphi) \sigma}{\sin \varphi \cos \varphi(Y+X)+Y\left(\cos ^{2} \varphi-\sin ^{2} \varphi\right)} \\
\sigma_{Y}=\frac{Y}{C} \frac{\sqrt{2}}{B} \frac{(X \sin \varphi+Y \cos \varphi)^{2} \sigma}{\sin \varphi \cos \varphi(Y+X)+Y\left(\cos ^{2} \varphi-\sin ^{2} \varphi\right)}
\end{gathered}
$$

\section{Case 3: Point A lies on the camera axis (CA).}

According to Figure 3

$$
\begin{gathered}
\frac{x}{C}=0.0 \\
\frac{x^{-}}{C}=\tan \alpha=\tan \varphi
\end{gathered}
$$

Substituting the values of $\left(x^{-} / C\right)$ and $(x / C)$ from Equations (27) and (28) into Equations (8) and (9) one finds that, the accuracy of the image coordinates of any point $\mathrm{A}$ lies on the camera axis (CA) are :

$$
\begin{aligned}
& \sigma_{x}=\left(1+\tan ^{2} \varphi\right) \\
& \sigma_{y}=\sec \varphi
\end{aligned}
$$

Substituting the values of $\sigma_{\mathrm{x}}$ and $\sigma_{\mathrm{y}}$ from equations 29 and 30 into equations $12 ; 13$ and 14 one get:

$$
\begin{aligned}
& \sigma_{X}=\frac{Y}{C}\left(1+\tan ^{2} \varphi\right) \quad \sigma \\
& \sigma_{Z}=\left(\frac{Y}{C}\right) \sec \varphi \sigma \\
& \sigma_{Y}=\frac{Y}{C} \frac{Y}{B} \sqrt{2} \quad\left(1+\tan ^{2} \varphi\right) \sigma
\end{aligned}
$$


THE AVERAGE ACCURACIES $\sigma_{X} ; \sigma_{Y}$ and $\sigma_{Z}$ OF THREEDIMENSIONAL OBJECTS

One can not expect the distribution of the ground points in each plane, Accordingly, the values $\sigma_{X} ; \sigma_{Y}$ and $\sigma_{Z}$ can be calculated in analytical photogrammetry using the numerical integration of $\sigma_{\mathrm{Xi}} ; \sigma_{\mathrm{Yi}}$ and $\sigma_{\mathrm{Zi}}$ and this can take the following form:

Case 1:

$$
\begin{gathered}
\sigma_{X}=\frac{1}{n} \sum_{i=1}^{n}\left[\frac{1}{C} \frac{1}{Y}(X \sin \varphi+Y \cos \varphi)_{\sigma}^{2}\right](34) \\
\sigma_{Z}=\frac{1}{n} \sum_{i=1}^{n}\left[\frac{1}{C}(X \sin \varphi+Y \cos \varphi)_{\sigma}\right] \\
\sigma_{Y}=\frac{1}{n} \sum_{i=1}^{n}\left[\frac{1}{C} \frac{1}{B} \sqrt{2}(X \sin \varphi+Y \cos \varphi)_{\sigma}^{2}\right](36)
\end{gathered}
$$

Case 2:

$$
\begin{aligned}
\sigma_{X} & =\frac{1}{n} \sum_{i=1}^{n}\left[\frac{1}{C} \frac{(X \sin \varphi+Y \cos \varphi)^{2} \sigma}{\sin \varphi \cos \varphi(Y+X)+Y\left(\cos ^{2} \varphi-\sin ^{2} \varphi\right)}\right](37) \\
\sigma_{Z} & =\frac{1}{n} \sum_{i=1}^{n}\left[\frac{Y}{C} \frac{(X \sin \varphi+Y \cos \varphi) \sigma}{\sin \varphi \cos \varphi(Y+X)+Y\left(\cos ^{2} \varphi-\sin ^{2} \varphi\right)}\right](38) \\
\sigma_{Y} & =\frac{1}{n} \sum_{i=1}^{n}\left[\frac{Y}{C} \frac{\sqrt{2}}{B} \frac{(X \sin \varphi+Y \cos \varphi)^{2} \sigma}{\sin \varphi \cos \varphi(Y+X)+Y\left(\cos ^{2} \varphi-\sin ^{2} \varphi\right)}\right](
\end{aligned}
$$

\section{Case 3:}

$$
\begin{aligned}
& \sigma_{X}=\frac{1}{n} \sum_{i=1}^{n}\left[\frac{Y}{C}\left(1+\tan ^{2} \varphi\right) \quad \sigma \quad\right](40) \\
& \sigma_{Z}=\frac{1}{n} \sum_{i=1}^{n}\left[\left(\frac{Y}{C}\right) \sec \varphi \sigma\right] \\
& \sigma_{Y}=\frac{1}{n} \sum_{i=1}^{n}\left[\frac{Y}{C} \frac{Y}{B} \sqrt{2} \quad\left(1+\tan ^{2} \varphi\right) \sigma\right]
\end{aligned}
$$

To determine the positions of points ( case 1, case2 and case 3 ) and to compute the average values of $\sigma_{\mathrm{Xi}} ; \quad \sigma_{\mathrm{Yi}}$ and $\sigma_{\mathrm{Zi}}$ from numerical integration of the new Formulas ( NEF) and those obtained from the present formulas ( PRF), a BASIC computer program was designed in this research work. The computed values of $\sigma_{\mathrm{Xi}} ; \sigma_{\mathrm{Yi}}$ and $\sigma_{\mathrm{Zi}}$ obtained from the numerical integration of the new 
formulas (NEF) (Equations 34- 42), from present formulas (PRF) and from simulation are given in Table 1

TABLE 1. COMPARIZON BETWEEN $\sigma_{\mathrm{X}}, \sigma_{\mathrm{Y}}$ and $\sigma_{\mathrm{Z}}$ COMPUTED FROM SIMULATION (SIM ), NEW FORMULAS (NEF) AND PRESENT FORMULAS (PRF)

\begin{tabular}{|c|c|c|c|c|c|c|c|c|c|}
\hline Pt. & \multicolumn{3}{|c|}{$\sigma_{\mathrm{X}}$} & \multicolumn{3}{|c|}{$\sigma_{\mathrm{Z}}$} & \multicolumn{3}{|c|}{$\sigma_{Y}$} \\
\hline & SIM & $\mathrm{NEF}$ & PRF & SIM & NEF & PRF & SIM & NEF & PRF \\
\hline 1 & 2.70 & 3.3 & 1.8 & 2.6 & 3.8 & 1.8 & 5.1 & 5.9 & 3.2 \\
\hline 2 & 3.2 & 3.2 & 1.8 & 3.9 & 3.8 & 1.9 & 5.6 & 5.9 & 3.2 \\
\hline 3 & 3.4 & 3.4 & 1.9 & 4.0 & 3.9 & 1.8 & 6.1 & 6.3 & 3.6 \\
\hline 4 & 3.3 & 3.4 & 1.6 & 3.9 & 3.8 & 1.7 & 6.0 & 6.1 & 3.4 \\
\hline 5 & 3.6 & 3.6 & 2.2 & 4.0 & 3.9 & 2.1 & 6.5 & 6.6 & 3.8 \\
\hline 6 & 3.4 & 3.6 & 1.9 & 4.1 & 3.9 & 2.1 & 6.4 & 6.5 & 3.6 \\
\hline 7 & 2.1 & 3.6 & 1.8 & 4.0 & 3.9 & 1.9 & 6.3 & 6.5 & 3.6 \\
\hline 8 & 3.8 & 3.6 & 2.1 & 3.8 & 3.8 & 2.1 & 6.3 & 6.4 & 3.7 \\
\hline 9 & 3.8 & 3.8 & 1.7 & 4.2 & 4.0 & 1.7 & 7.0 & 7.1 & 4.1 \\
\hline 10 & 3.0 & 3.8 & 1.8 & 3.6 & 3.9 & 1.9 & 5.5 & 6.7 & 4.1 \\
\hline 11 & 5.5 & 6.1 & 2.1 & 6.0 & 6.2 & 2.1 & 16.5 & 17.5 & 8.8 \\
\hline 12 & 3.7 & 3.9 & 1.6 & 3.9 & 3.9 & 1.8 & 7.2 & 7.2 & 3.9 \\
\hline 13 & 3.9 & 3.9 & 1.5 & 4.1 & 4.0 & 1.7 & 7.0 & 7.3 & 3.8 \\
\hline 14 & 3.8 & 3.9 & 1.6 & 4.7 & 4.0 & 1.4 & 6.6 & 7.3 & 3.8 \\
\hline 15 & 5.4 & 5.5 & 1.8 & 5.8 & 5.6 & 1.9 & 11.6 & 13.9 & 6.2 \\
\hline 16 & 4.0 & 4.0 & 2.1 & 4.1 & 4.0 & 2.1 & 7.1 & 7.4 & 4.2 \\
\hline 17 & 4.0 & 4.1 & 1.9 & 4.2 & 4.0 & 1.9 & 7.6 & 7.7 & 4.2 \\
\hline 18 & 1.58 & 1.57 & 0.8 & 1.13 & 1.12 & 0.8 & 0.29 & 0.49 & 0.8 \\
\hline 19 & 4.0 & 4.3 & 2.1 & 4.2 & 4.1 & 2.1 & 6.2 & 6.5 & 3.3 \\
\hline 20 & 4.1 & 4.2 & 2.3 & 5.1 & 4.1 & 2.3 & 7.6 & 7.7 & 4.6 \\
\hline 21 & 5.4 & 5.8 & 1.8 & 5.7 & 5.6 & 1.9 & 14.5 & 14.8 & 6.8 \\
\hline 22 & 5.3 & 5.2 & 2.3 & 4.9 & 4.9 & 2.3 & 9.4 & 9.6 & 4.2 \\
\hline 23 & 6.1 & 6.5 & 1.9 & 5.6 & 6.4 & 1.9 & 15.8 & 18.6 & 8.5 \\
\hline 24 & 5.1 & 5.2 & 2.1 & 5.8 & 4.9 & 2.1 & 9.1 & 9.5 & 4.8 \\
\hline 25 & 5.3 & 5.3 & 2.1 & 5.1 & 4.9 & 2.1 & 9.6 & 9.6 & 4.9 \\
\hline 26 & 3.9 & 4.7 & 2.3 & 3.9 & 4.2 & 2.1 & 5.9 & 7.1 & 3.2 \\
\hline 27 & 5.0 & 5.4 & 1.9 & 5.1 & 5.0 & 1.8 & 9.2 & 9.9 & 4.4 \\
\hline 28 & 4.6 & 4.9 & 2.1 & 4.4 & 4.3 & 2.1 & 7.1 & 7.4 & 4.1 \\
\hline
\end{tabular}




\section{CONCLUSIONS}

From Table 1 one can conclude that :

- The proposed formulas are conform with the simulation technique and give better values of $\sigma_{x} ; \sigma_{y}$ and $\sigma_{z}$ when compared to the present formulas.

-The proposed formulas give good results at the center or at the edge of the photograph.

- One can use the proposed formula to determine the approprite positions right on the field by computing the average values of $\sigma_{x} ; \sigma_{y}$ and $\sigma_{z}$ which can be estimated from the numerical integration of the formulas in the three cases.

-For the simulation technique one need a personal computer but for the proposed formula one need only a small calculator .

For all these reasons one can suggested to use the proposed formulas instead of the exisiting formulas and the simulation method.

\section{REFERENCES}

[1]Abdel-Aziz,Y.I.,andKarara,H.M.,1973, "Photogrammetric Potentional of Non-Metric Cameras," Photogrammetry Series No:35, University of Illinoise, Urbana.Illinois.

[2]Abdel-Aziz,Y.I.,1974, Expected Accuracy of Convergent Photography, Photogrammetric Engineering and Remote Sensing, Vol.40, No.11 November 1974.

[3]Abdel-Aziz Y.I.,1982, Accuracy of the normal case of close -range photogrammetry, Photogrammetric Engineering and Remote Sensing,Vol.48,No.2, February 1982,pp. 207-213.

[4]Donald L. Light,1992, The New Camera Calibration System At the U.S. Geological Survey Photogrammetric Engineering and Remote Sensing, Vol.58, No.2 February 1992,pp.185-188.

[5]Franz W.Leberl and Hubert Ranzinger 1997 ,Registration of Digitized Aerial Photography with a Digital Map Data Base, DIBAGReport No.6, Institute for Image Processing and Computer Graphics, Tecnical University and Graz Research Center.

[6]Hottier,PH.(1976) Accuracy of Close Range Analytical restitutions: practical experiments and prediction.PE, vol.42, No.3 march 1976, pp345-375.

[7]Karara,H.M.,1966,"Stereometric Systems of High Precision," Civil Engineering Studies, Photogrammetry Series No.15, University of Illinoise, Urbana.Illinois.

[8]Regensburger,K.(1990) Photogrammetrie, Anwendungen in Wissenschaft und Technik.1990: Berlin VEB verlag fur Bauwesen. 


\section{الإقة المثوقعة فى الصور المثقاربة

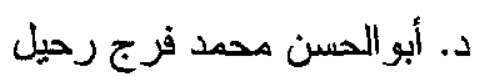 كلية الهنسة - جامعة المنوفية - شبين الكوم}

ليس هنالك شك فى زيادة واتساع تطبيقات الفوتوجر امترى وتتعد الصور المثتاربـــة

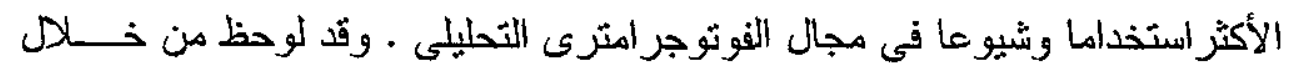

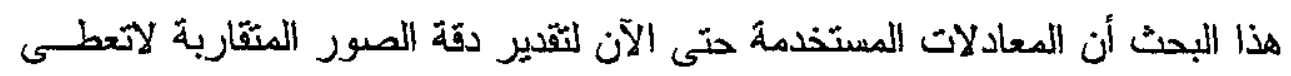

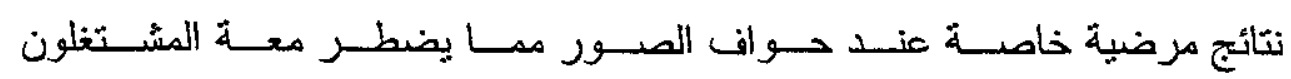
بالفوتوجر امترى لاستخدام طريقة المحاكاة رغم عيوبها الكثيرة لنقدير الدقة .

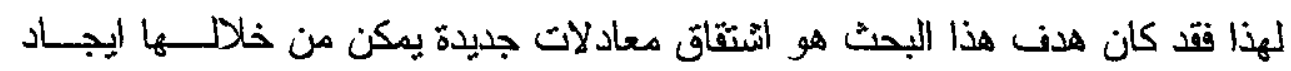

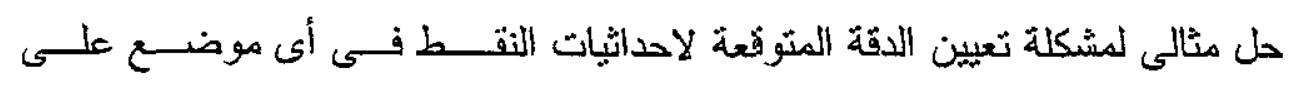

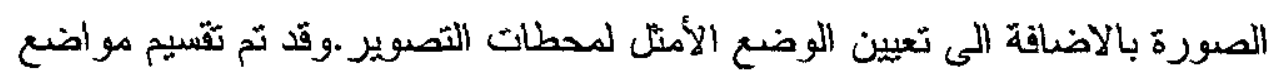

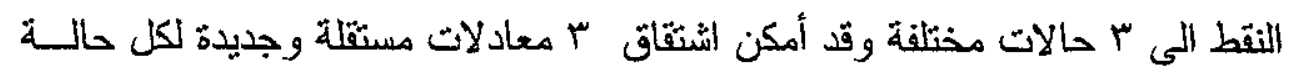

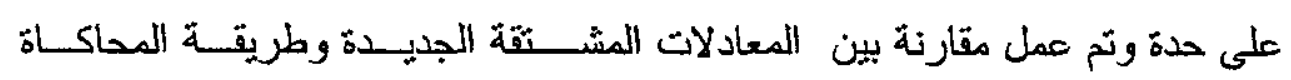

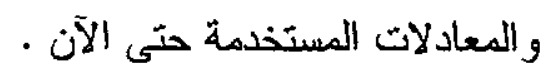

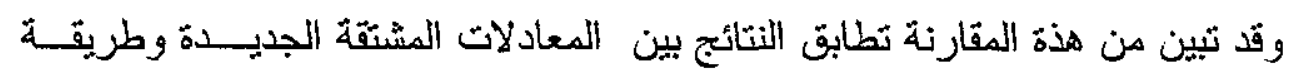

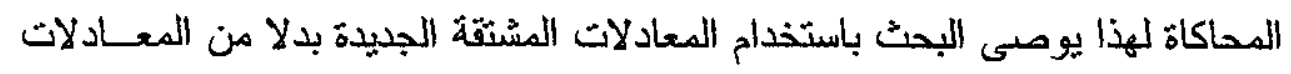
المستخدمة حاليا لما لهذة المعادلات الجديدة من مميز ات كثيرة. 